\title{
Opioid prescribing patterns among medical providers in the United States, 2003-17: retrospective, observational study
}

\author{
Mathew V Kiang, ${ }^{1,2}$ Keith Humphreys, ${ }^{2,3}$ Mark R Cullen, ${ }^{1}$ Sanjay Basu ${ }^{4,5,6}$
}

${ }^{1}$ Center for Population Health

Sciences, Stanford University

School of Medicine, 1701 Page Mill Road, Palo Alto, CA 94304 , USA

${ }^{2}$ Department of Psychiatry and Behavioral Sciences, Stanford University School of Medicine,

Palo Alto, CA, USA

${ }^{3}$ Palo Alto VA Health Care System, Palo Alto, CA, USA

${ }^{4}$ Center for Primary Care, Harvard Medical School, Boston, MA, USA

${ }^{5}$ Research and Analytics, Collective Health, San Francisco, CA, USA

${ }^{6}$ School of Public Health, Imperial College, London, UK Correspondence to: M V Kiang mkiang@stanford.edu (or @mathewkiang on Twitter: ORCID 0000-0001-9198-150X) Additional material is published online only. To view please visit the journal online.

Cite this as: $B M J$ 2020;368:16968 http://dx.doi.org/10.1136/bmj.16968

Accepted: 3 December 2019

\section{Abstract}

OBJECTIVE

To examine the distribution and patterns of opioid prescribing in the United States.

DESIGN

Retrospective, observational study.

SETTING

National private insurer covering all 50 US states and Washington DC.

\section{PARTICIPANTS}

An annual average of 669495 providers prescribing 8.9 million opioid prescriptions to 3.9 million patients from 2003 through 2017.

\section{MAIN OUTCOME MEASURES}

Standardized doses of opioids in morphine milligram equivalents (MMEs) and number of opioid prescriptions.

\section{RESULTS}

In 2017 , the top $1 \%$ of providers accounted for $49 \%$ of all opioid doses and $27 \%$ of all opioid prescriptions. In absolute terms, the top $1 \%$ of providers prescribed an average of 748000 MMEs-nearly 1000 times more than the middle $1 \%$. At least half of all providers in the top $1 \%$ in one year were also in the top $1 \%$ in adjacent years. More than two fifths of all prescriptions written by the top $1 \%$ of providers were for more than 50 MMEs a day and over four fifths were for longer than seven days. In contrast, prescriptions written by the bottom $99 \%$ of providers were below these thresholds, with $86 \%$ of prescriptions for less than 50 MMEs a day and $71 \%$ for fewer than seven days. Providers prescribing high amounts of opioids and patients receiving high amounts of opioids persisted over time, with over half of both appearing in adjacent years.

\section{WHAT IS ALREADY KNOWN ON THIS TOPIC}

Opioid prescribing remains far higher in the US than in other countries, despite efforts to reduce inappropriate opioid prescribing

Previous studies have noted that opioid prescribing in the US is skewed; however, the extent of this skewness in a geographically diverse and demographically representative population has not been well established

\section{WHAT THIS STUDY ADDS}

This study found that about $1 \%$ of providers account for nearly half of all opioid doses and one quarter of all opioid prescriptions

The results suggest that rather than impose rigid thresholds on most providers, who generally prescribe opioids safely, interventions should focus on the top $1 \%$ of providers and their patients

The results also suggest that interventions should focus on improving patient care, managing patients with complex pain, and reducing comorbidities

\section{CONCLUSIONS}

Most prescriptions written by the majority of providers are under the recommended thresholds, suggesting that most US providers are careful in their prescribing. Interventions focusing on this group of providers are unlikely to effect beneficial change and could induce unnecessary burden. A large proportion of providers have established relationships with their patients over multiple years. Interventions to reduce inappropriate opioid prescribing should be focused on improving patient care, management of patients with complex pain, and reducing comorbidities rather than seeking to enforce a threshold for prescribing.

\section{Introduction}

From 1999 to 2010, opioid prescribing in the US quadrupled, ${ }^{1}$ reaching a per capita level well beyond that of any other nation. ${ }^{2}$ The effectiveness of long term opioid treatment for management of chronic pain is unclear, ${ }^{34}$ but opioids remain an essential tool for medical providers. Inappropriate opioid prescribing can lead to diversion of, addiction to, and overdose from prescription opioids, ${ }^{5-9}$ contributing to an epidemic of opioid related deaths in recent years. ${ }^{10}$ Promoting cautious, scientifically justified, opioid prescribing has become a leading goal of policy makers in both government and healthcare. ${ }^{11-14}$

Strategies to reduce inappropriate opioid prescribing range from broad policies or guidelines targeted at all medical providers to narrow interventions focused on providers prescribing high amounts of opioids. Broad strategies include improving medical school education curriculums and compulsory education of providers, ${ }^{15}$ mandating the use of state level prescription drug monitoring programs, creating national clinical guidelines, ${ }^{16}$ and lowering the default opioid dose in electronic health records. ${ }^{17-20}$ Targeted interventions typically focus on providers who prescribe opioids above a specified threshold. A common targeted intervention is the creation of "pill mill" laws, which typically require documentation of medical examinations and follow-up visits before and after the prescribing of opioids, mandatory registration of clinics with the state, or physician ownership of pain clinics. $^{21-25}$

Targeted interventions do not require new laws. For example, one national pharmacy chain identified 42 providers, out of nearly one million, who prescribed excessive levels of opioids (eg, 98th centile for volume, 95th centile for proportion, and had a high number of patients who paid cash) and requested documentation justifying their level of prescribing. Of the 42 providers contacted, only six responded with medically justified reasons. The remaining 36 who did not respond or did 
not provide justification were no longer allowed to have their prescriptions filled at this national pharmacy chain. ${ }^{26}$ Alternatively, "light touch" interventions have been proposed, such as sending letters to providers whose prescribing patterns are higher than those of their peers ${ }^{27}$ or notifying those providers whose patients have had a fatal overdose. ${ }^{28}$

Both broad strategies and targeted interventions are important for reducing inappropriate prescribing. However, effective opioid prescribing is nuanced, pain management is often complex, and there can be legitimate clinical reasons for a provider prescribing opioids in excess of recommended thresholds. Strategies to reduce prescribing can result in unintended consequences, such as misapplication, which could result in poor care or sudden involuntary discontinuation of opioids. ${ }^{29}$ Further, institutions and agencies responsible for enforcing or implementing these strategies may have limited resources and require additional information to prioritize strategies.

For these reasons, data driven policy demands a careful examination of opioid prescribing patterns over a broader range of time, geography, and population. Previous studies directly examining the distribution of opioid prescriptions among providers have been limited to geographically or demographically narrow samples of providers or patients, over shorter periods. ${ }^{3031}$ This study seeks to fill these gaps, provide clarity to policy makers, and assist institutions with efforts to prioritize reduction. We examined a repository of prescription claims from a large, national insurance provider covering more than 60 million unique individuals from 2003 to 2017 . The insured population includes all 50 US states and Washington DC and is of similar age and sex to the US general population. Using these data, we identified whether opioids are roughly equally prescribed across US medical providers or disproportionately prescribed by a small subset of providers, how prescribing patterns have changed over time, the extent to which top prescribers and top receiving patients persisted over time, and the relationship between high prescribing and top receiving patients.

\section{Methods}

Data

From a total of 2.5 billion outpatient prescription claims, we identified 134 million opioid prescriptions in the Optum Clinformatics Data Mart Database (Optum; 2003-17), a large deidentified database from a national private insurance provider. Prescriptions that were filled but not dispensed (that is, not received by the patient) were excluded from the analysis to prevent double counting, which might happen, for example, if a prescription was transferred to, and dispensed by, a different pharmacy. Prescription level data included hashed patient identifiers, hashed provider identifiers (that is, Drug Enforcement Agency and National Provider Identifier), National Drug Code, quantity, number of days of supply, and date of transaction. In addition, we used the member, provider, and outpatient diagnostic files from 2003 to 2017. An Optum affiliate company assigns providers into one of 7106 categories, which are grouped into 308 specialties. We merged provider specialties into 16 larger, mutually exclusive categories: addiction medicine, anesthesiology, surgery, critical care, dentistry, emergency medicine, family medicine, general medicine, hospice care, internal medicine, obstetrics/gynecology, pediatrics, physical or pain medicine and rehabilitation, pharmacy, unknown, and all others. For female enrollees the median age was 34 years (50.5\%, interquartile range $19-52$ years) and for male enrollees 33 years (49.5\%, 18-49 years). The age and sex distribution of enrollees in the Optum database closely resembles that of the general US population (supplementary table S1).

\section{Defining drugs of interest}

We followed US Centers for Disease Control and Prevention (CDC) recommendations when selecting the most appropriate opioids for our analysis. ${ }^{32}$ Specifically, we excluded buprenorphine products prescribed as treatment for opioid use disorder, drugs typically not used in outpatient settings (eg, fentanyl in solution), cough and cold formulations, and injectable or intravenous opioids.

As with any system, some unequal distribution is expected, ${ }^{33}$ due, for example, to differences in provider preferences, ${ }^{34}$ provider location, ${ }^{35}$ provider specialties, ${ }^{36} 37$ or provider perceptions. ${ }^{38}$ Thus to facilitate comparison, we tabulated eight benzodiazepines used to treat anxiety, panic disorder, seizures, alcohol withdrawal symptoms, and muscle spasms: alprazolam, chlordiazepoxide, clonazepam, diazepam, lorazepam, oxazepam, temazepam, and triazolam. Similar to opioids, benzodiazepines have highly variable prescribing patterns and a wide range of doses and therapeutic uses, including episodic use. A full list of National Drug Codes for each drug can be found in the online code repository (see supplementary materials section 1 ).

\section{Quantities of interest}

We estimated two primary quantities of interest for each drug and year, for each provider: dose and number of prescriptions. In addition, we estimated three secondary quantities of interest: daily dose, dose for each patient, and dose for each prescription. Opioid doses were standardized to morphine milligram equivalents (MMEs) using National Drug Code specific conversion factors. ${ }^{32}$ Benzodiazepine doses were standardized to lorazepam milligram equivalents. ${ }^{39-43}$ Dose was calculated as the quantity $\times$ drug strength $\times$ conversion factor. For each provider, we took the total number of opioid or benzodiazepine prescriptions across all patients. The daily dose was calculated as the dose divided by the number of days supply for each prescription. The dose for each patient was calculated as the total dose divided by the number of unique patients who received an opioid or benzodiazepine prescription from each provider separately (that is, a patient receiving multiple opioid prescriptions from 
multiple providers counts as one unique patient for each provider). The dose for each prescription was calculated as the total dose divided by the total number of opioid or benzodiazepine prescriptions for each provider.

\section{Estimating disproportionate levels of quantities of interest}

To describe the distribution of the above quantities of interest, we partitioned providers, patients, and provider-patient pairs with at least one opioid prescription into 100 equally sized groups. The groups were separated according to centiles of the volume of opioids prescribed, received, and transacted, respectively. We then compared the prescribing patterns of the top centile group of providers (that is, top $1 \%$ of providers) with the median (50th) centile group. Additionally, we quantified the entire distribution of prescribing using the Gini coefficient-a formal summary measure of global inequality. ${ }^{44} 45$ Supplementary materials section 2 presents the Gini coefficient results.

\section{Defining recent primary diagnoses and previous diagnosis of malignant cancer}

For the top centile of patients, we tabulated the recent primary diagnoses of their visits to the provider. In the Optum dataset, patient prescriptions and diagnoses are not directly linked. Therefore, we conservatively defined a recent primary diagnosis as any primary diagnosis that occurred in the same year or in the year before a patient appeared in the top centile of patients receiving opioids. We collapsed these recent primary diagnosis ICD-9 and ICD-10 codes (international classification of diseases, ninth and 10th revisions, respectively) into general medical categories using the Clinical Classifications Software to ICD crosswalk provided by the Agency for Healthcare Research and Quality. ${ }^{46}$ We defined a previous diagnosis of malignant cancer as the presence of an ICD code for any malignant cancer in the current year or any previous year in which the patient appeared in the top centile of patients receiving opioids (supplementary materials section 3). This definition conservatively includes cancers in remission.

\section{Sensitivity analyses}

We conducted several sensitivity analyses. To test the robustness of our results to miscoding or extreme prescribing outliers, we repeated our analyses with different levels of upper truncation. Specifically, we removed the upper $0.01 \%, 0.05 \%, 1.0 \%, 2.5 \%$, $5.0 \%$, and $10 \%$ of prescribers for each year and drug type. To test the robustness of our results to a high prevalence of low activity prescribers, we repeated our analyses with different levels of prescribing activity. Specifically, we removed all providers who prescribed fewer than 3, 6, 12, 24, 50, 100, and 200 prescriptions a year. To test the robustness of our results to the subset of opioids used, we repeated our analyses using only opioids designated as schedule 2 under the Controlled Substances Act. Schedule 2 controlled substances are drugs with an accepted medical use but with a high potential for misuse and that could lead to psychological or physical dependence. To test the sensitivity of the Gini coefficient, we estimated several alternative measures of inequality: RicciSchutz, Atkinson, Theil, and generalized entropy (supplementary materials section 4). ${ }^{47}$ Finally, in addition to standardized benzodiazepine and opioid doses, we analyzed the prescribing patterns for 18 individual drugs, with a range of therapeutic uses (supplementary materials section 5). In all instances, our substantive conclusions remained the same. Results for additional analyses are available using an interactive results viewer (supplementary materials section 6).

\section{Patient and public involvement}

No patients were involved in setting the research question or the outcome measures, nor were they involved in developing plans for design or implementation of the study. No patients were asked to advise about interpretation or writing up of results.

\section{Results}

\section{Disproportionate prescribing patterns}

Between 2003 and 2017, an average of 8.2 billion MMEs were prescribed by 669495 providers to 3.9 million patients a year (supplementary table S2 and figs S1 and S2). These prescribing patterns correspond to an average of more than 700 MMEs daily for each provider, more than 120 MMEs daily for each patient, more than 50 MMEs daily for each prescription, and 8.9 million opioid prescriptions a year. These averages, however, mask highly skewed distributions.

In 2017, the top centile of providers prescribed $49 \%$ of all opioid doses and $27 \%$ of benzodiazepine doses (fig 1). The distribution of prescriptions was comparably disproportionate, with the top centile of providers accounting for $27 \%$ of opioid prescriptions and $19 \%$ of benzodiazepine prescriptions (fig 1). These disproportionate prescribing patterns persisted after accounting for prescription length, number of patients for each provider, and number of prescriptions for each provider (supplementary materials section 6).

The disproportionately high prescribing of the top centile of providers has been stable since 2008 (fig 2) and is consistent across states (supplementary figs S3 and S4). Between 2003 and 2017, the top centile of providers prescribed between 42\% (2005) and 49\% (2008) of opioid doses and accounted for between $18 \%$ (2004) and 27\% (2017) of all opioid prescriptions (fig 2). A lower level of disproportionate prescribing was observed for benzodiazepines. Specifically, the top centile of providers prescribed between 23\% (2006) and 29\% (2008) of benzodiazepine doses and accounted for between 17\% (2006) and 21\% (2008) of all benzodiazepine prescriptions (fig 2). The type of opioid or benzodiazepine prescribed by the top centile of providers was similar to that prescribed by all providers, with the exception of 

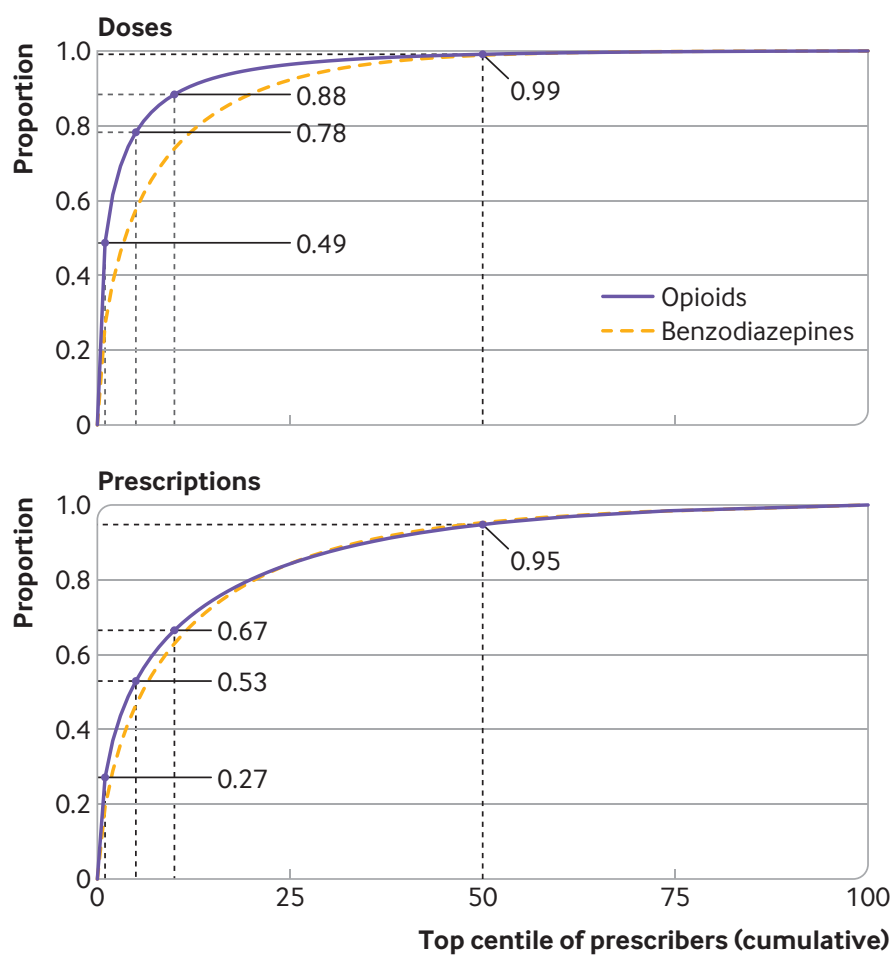

Fig 1 | Distribution of standardized doses and prescriptions for opioids and benzodiazepines across all providers in 2017 . Dotted lines show top $1 \%, 5 \%, 10 \%$, and $50 \%$ of providers for each type of drug along with proportion of opioids distributed by each of these groups guidelines, with $42 \%$ to $49 \%$ of their prescriptions being a higher dose than recommended. Similarly, $81 \%$ to $98 \%$ of prescriptions written by the top centile of providers were for more than seven days. Despite the disproportionate prescribing of the top centile, most providers are prescribing within these guidelines. In each year from 2003 to 2017 , at least $86 \%$ of prescriptions by the bottom $99 \%$ of providers were less than 50 MMEs a day. Similarly, at least $71 \%$ of prescriptions by the bottom $99 \%$ of providers were for fewer than seven days (supplementary table S3 and fig S8).

\section{Characteristics of the top centile group of providers and patients}

More than half of the top centile of opioid prescribers are in family medicine (24\%), physical or pain medicine and rehabilitation (14\%), anesthesiology (14\%), or internal medicine (13\%); about one third are classified as other $(17 \%)$ or unknown (14\%). Physical or pain medicine and rehabilitation and anesthesiology are the most over-represented specialties in the top centile, with each accounting for just $1 \%$ of providers across all centiles. Family medicine and internal medicine are slightly over-represented in the top centile, and account for just $13 \%$ and $8 \%$ of providers, respectively, across all centiles. Hospice and critical care specialists accounted for less than $1 \%$ of both the top centile group and across all providers (supplementary fig S9).

Among the top centile of patients receiving opioids, the most common recent primary diagnostic indication was a "back problem," followed by "other connective tissue disease," "other aftercare," "other nervous system disorders," and "unclassified" (supplementary table S4). Less than $20 \%$ of patients in the top centile receiving opioids had any diagnosis of malignant cancer during the study period (including patients with cancers currently in remission) (supplementary table S5).

In 2017, the top centile of patients receiving opioids was prescribed 2.87 billion MMEs (28\% of all opioids transacted), $70 \%$ of which originated from the top centile of providers (fig 3). This level of disproportionate transactions between provider and patient was not observed for benzodiazepines. Specifically, the top centile of patients receiving benzodiazepine were prescribed only $12 \%$ of all benzodiazepine doses, about half of which originated from the top centile of providers (fig 3). As a result of the disproportionate prescribing of the top centile, the bottom 90 centiles of providers accounted for only $12 \%$ of opioids transacted compared with $26 \%$ for benzodiazepine. Similarly, as a result of the highly linked nature of the top centile of providers and patients, the majority of opioids (72\%) originating from the bottom 90 centiles of providers were received by the bottom 90 centiles of patients.

The top centile providers, patients, and providerpatient pairs persisted over time (fig 4). Specifically, between $54 \%$ and $73 \%$ of the top of providers in any year were also in the top centile in the previous year, with modest reduction in more distant years. The pattern of high overlap in adjacent years was also 

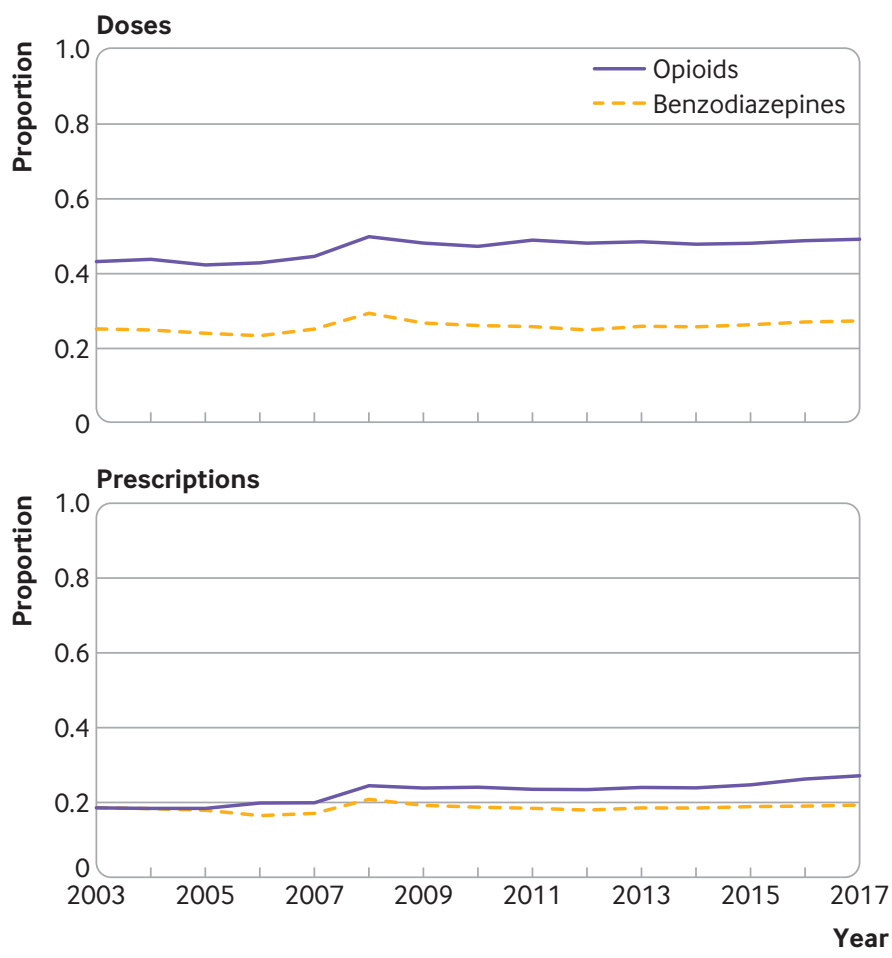

Fig 2 | Proportion of standardized doses and prescriptions prescribed by top $1 \%$ of providers for opioids and benzodiazepines, 2003-17

seen, though attenuated, among top centile patients and provider-patient pairs (fig 4), suggesting that high opioid prescribing and receiving is stable over time and the top centile provider-patient pairs have established associations over multiple years (supplementary figs S10 and S11).

\section{Discussion}

Promotion of cautious, scientifically justified opioid prescribing is an important public health goal. The US has started to make progress towards this goal in recent years. ${ }^{48}$ Policy makers use broad, tailored strategies to reduce inappropriate opioid prescribing. To understand the implications of these strategies, including unintended consequences, and to prioritize their implementation, requires a nuanced description of opioid prescribing patterns. Skewed opioid prescribing has been found in previous studies, although to different extents. One study carried out a smaller examination of a single state and another, was a geographically dispersed examination of the older Medicare Part D population. ${ }^{30}{ }^{31}$ Our study extends these reports by examining a large, national sample with an age and sex distribution closely resembling that of the US general population. Additionally, this study adds detail by examining trends over time by provider specialty, underlying opioid type, and provider-patient linkages.

Our results clarify an important medical reality. Specifically, our results suggest that interventions targeted at high prescribing opioid providers should be prioritized for three reasons. First, most of the prescriptions written by the majority of providers are below recommended thresholds, suggesting that most US providers prescribe opioids carefully. Additional, potentially burdensome, constraints on this set of providers are unlikely to be beneficial. Because the majority of opioid prescriptions by this group are probably appropriate, any increase in restrictions is more likely to reduce appropriate use rather than prevent those of concern. Second, the top centile of providers is stable over time, which suggests that interventions targeted at this group will produce sustained results. Third, the top centile of providers accounts for a disproportionate number of patients receiving high amounts of opioids, and interventions targeted here will reach these high risk patients efficiently.

In addition, our results suggest that interventions must be nuanced and tailored. More than one quarter of providers in the top centile specialize in anesthesiology or physical or pain medicine and rehabilitation, two specialties in which additional training in clinically appropriate opioid prescribing is received. Further, a substantial fraction of the top centile of provider-patient pairs overlap in adjacent years, suggesting established relationships over many years. Extended relationships with patients could enable providers to have a better understanding of patients' needs and a readiness to taper doses. Lastly, the top centile of patients remains the same over time, which suggests that interventions based on improving patient care, management of patients with complex problems, and reducing comorbidities, rather than enforced interventions focused on rigid thresholds, are necessary to reduce demand for opioids.

\section{Limitations of this study}

Our analysis has important limitations. First, our data were obtained from a single national insurer comprising mostly employer based, privately insured patients and may not be fully generalisable to the whole of the US. Second, the prescription data included only dispensed prescriptions and not prescriptions that were written by providers but never received by patients; therefore, these data represent a potentially biased subset of provider prescribing patterns. Nevertheless, these data covered a patient population closely representative of theage and sex distribution of the general US population (supplementary table S1). Additionally, when our data were compared with a careful examination of opioid prescriptions in California, similar results were found (supplementary fig S12). Third,prescriptions attributed to one provider could have originated from care provided by a different provider (for example, primary care providers carrying out the treatment plan of a pain clinic). Fourth, opioids are a diverse set of drugs and standardizing doses into MMEs is necessary for comparisons; however, MMEs may not fully reflect the dependence, overdose, or mortality potential of different opioids. Sensitivity analyses using only schedule 2 opioids-those considered to have the highest potential for misuse-nevertheless 

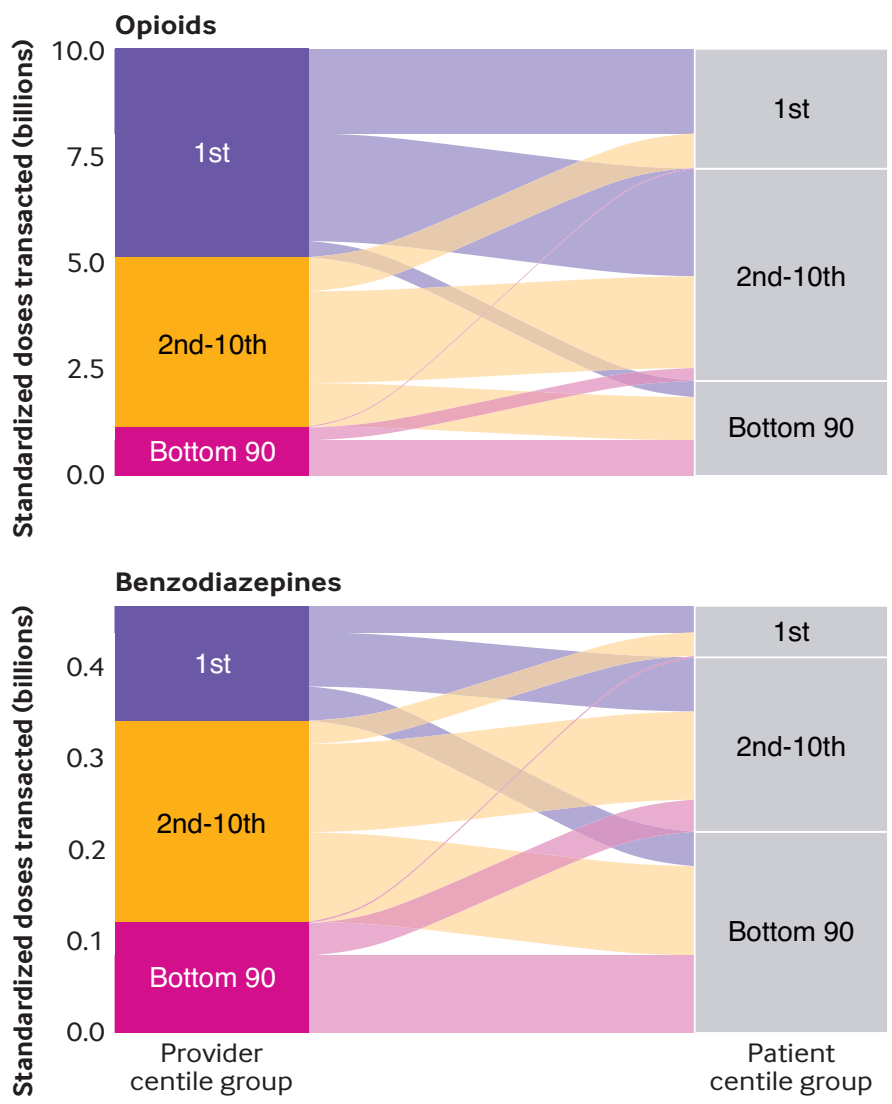

Fig 3 | Volume of opioid and benzodiazepine doses transacted between provider and patient centile groups, 2017

reached the same substantive conclusions. Similarly, benzodiazepines are diverse, and conversion factors for equipotency between different benzodiazepines have not been well studied. Importantly, our data did not allow us to assess the clinical appropriateness of any opioid or benzodiazepine prescription. Lastly, reducing inappropriate opioid prescribing is a worthwhile goal in and of itself; however, the opioid overdose crisis has shifted from prescription opioids to illicit opioids, ${ }^{10}{ }^{49}$ such as heroin and synthetic opioids, which have mortality rates that are higher than those of prescription opioids and are increasing more rapidly. Therefore, the effect of strategies to reduce inappropriate prescribing on the opioid overdose crisis at large is probably limited. The recent increase in deaths from illicit opioids is, in part, an unintended consequence of reducing the availability of medically prescribed opioids, which has led some opioid dependent patients to turn to the illicit marketplace. ${ }^{50}$

\section{Conclusions and policy implications}

Our study shows that in the claims database of a national private insurer, a small portion of providers account for a highly disproportionate proportion of opioids. Further, these providers persist over time and are often linked with patients receiving high amounts of opioids. A corollary of this finding is that most of the prescriptions written by the majority of providers are below recommended thresholds. Specialties with additional training in clinically appropriate opioid prescribing-for example, anesthesiology and physical or pain management and rehabilitation-are overrepresented in the top centile of opioid prescribing providers. Lastly, we found that a substantial proportion of provider-patient pairs are linked over time. These findings suggest that interventions promoting careful prescribing should be tailored for, and targeted at, the top centile of opioid prescribing providers and focus on providing supportive care of

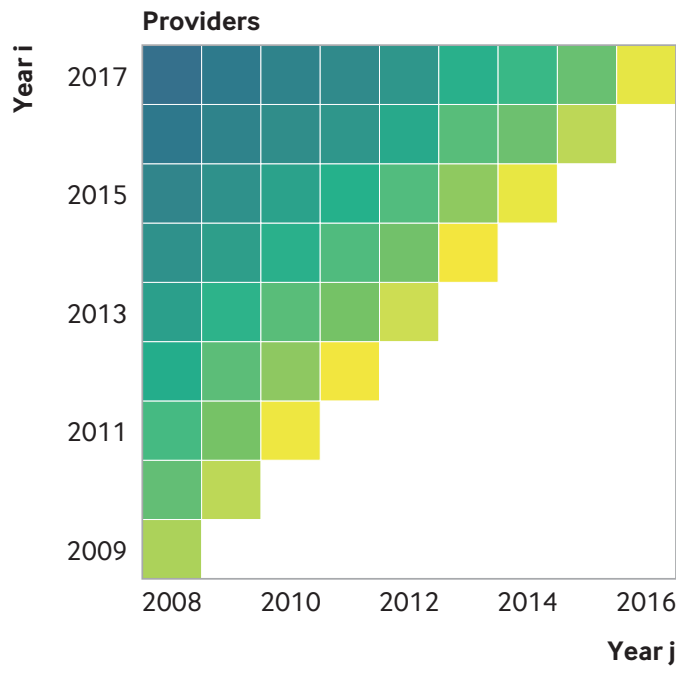

Patients

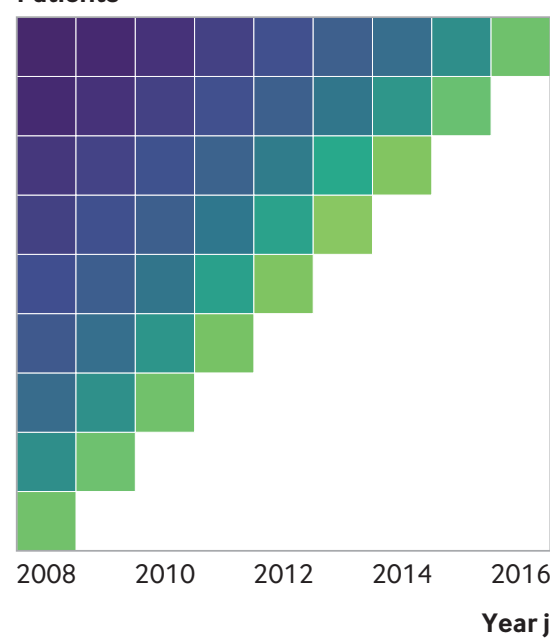

\section{Provider-patient pairs}

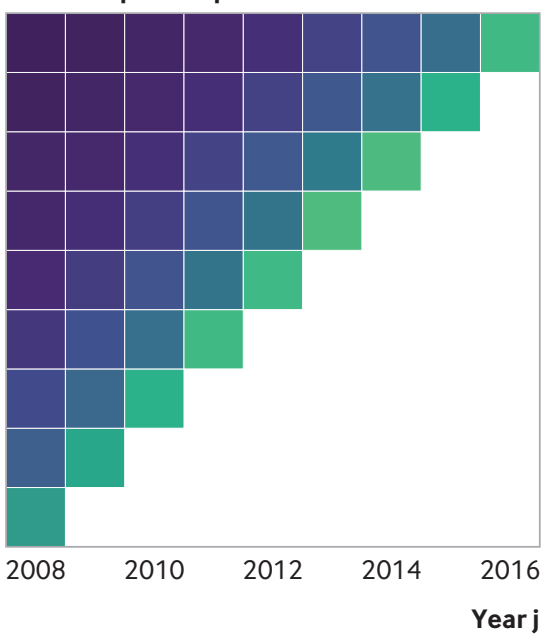

Proportion from year $i$ in year $j$

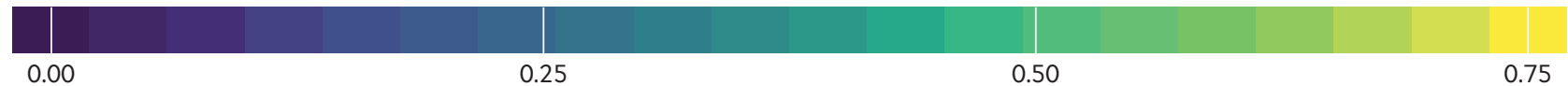

Fig 4 | Persistence of top centile of providers, patients, and provider-patient pairs over time. 
patients with complex problems rather than seeking to enforce a threshold for prescribing.

We thank Lesley Park, Emma Hallgren, Valerie Meausoone, and Isabella Chu at the Stanford Center for Population Health Sciences Data Core for technical assistance.

Contributors: MVK and SB conceptualized the paper. MVK analyzed the data with input from SB. All authors interpreted results. SB and MRC provided financial support for the data and computational resources. MVK, SB, and KH wrote the initial draft with all authors providing critical feedback and edits to subsequent revisions. All authors approved the final draft of the manuscript. MVK and SB are the guarantors. The corresponding author attests that all listed authors meet authorship criteria and that no others meeting the criteria have been omitted.

Funding: This study did not receive specific funding. MVK is supported in part by the National Institute on Drug Abuse of the National Institutes of Health (NIH) (T32 DA035165). MVK and SB were supported in part by the National Institute on Minority Health and Health Disparities of the NIH (DP2 MD010478). KH was supported by a senior research career scientist award from the Veterans Affairs Health Services Research and Development Service and a grant from Stanford Neurosciences Institute. Data access was provided by the Stanford Center for Population Health Sciences Data Core. The Population Health Sciences Data Core is supported by an NIH national center for advancing translational science clinical and translational science award (UL1 TR001085) and internal Stanford funding. The funders had no role in the study design, data collection and analysis, decision to publish, or preparation of the manuscript. The content is solely the responsibility of the authors and does not necessarily represent the official views of the funders, federal government, or any current or former employers of the authors. Data and analyses were conducted at Stanford University under a data use agreement between Stanford and Optum.

Competing interests: All authors have completed the ICMJE uniform disclosure form at www.icmje.org/coi_disclosure.pdf and declare: no support from any organization for the submitted work; SB reports grants from the US National Institutes of Health, US Department of Agriculture, and Robert Wood Johnson Foundation, and personal fees from Collective Health, KPMG, Research Triangle International, PLOS Medicine, and the New England Journal of Medicine, outside the submitted work; no financial relationships with any organizations that might have an interested in the submitted work in the previous three years; no other relationships or activities that could appear to have influenced the submitted work.

Ethical approval: This study was approved by the Stanford University institutional review board (PHS-40974).

Data sharing: Original, prescription level and diagnosis level data tied to individuals, providers, locations, and time are considered personally identifiable health information. These data cannot be shared owing to risks of breaching patient confidentiality. The authors have posted full reproducible code on the public GitHub repository associated with this project, such that researchers who engage in a data use agreement with Optum can use this code to reproduce or extend the analysis. Aggregated data are available to the extent allowed by a data use agreement. The repository is available at https://github.com/mkiang/disproportionate_prescribing (supplementary materials section 1).

The lead author (MVK) affirms that the manuscript is an honest, accurate, and transparent account of the study being reported; that no important aspects of the study have been omitted; and that any discrepancies from the study as planned have been explained. Dissemination to participants and related patient and public communities: There are no plans to disseminate the results of the research to study participants or the relevant patient community.

This is an Open Access article distributed in accordance with the Creative Commons Attribution Non Commercial (CC BY-NC 4.0) license, which permits others to distribute, remix, adapt, build upon this work non-commercially, and license their derivative works on different terms, provided the original work is properly cited and the use is noncommercial. See: http://creativecommons.org/licenses/by-nc/4.0/.

1 Centers for Disease Control and Prevention. Vital Signs: Overdoses of Prescription Opioid Pain Relievers United States, 1999--2008. MMWR Morb Mortal Wkly Rep 2011;60:1487-92.

2 Humphreys K. Avoiding globalisation of the prescription opioid epidemic. Lancet 2017;390:437-9. doi:10.1016/S01406736(17)31918-9
3 Von Korff M, Kolodny A, Deyo RA, Chou R. Long-term opioid therapy reconsidered. Ann Intern Med 2011;155:325-8. doi:10.7326/0003 4819-155-5-201109060-00011

4 Chou R, Deyo R, Devine B, et al. The effectiveness and risks of longterm opioid treatment of chronic pain. Evid Rep Technol Assess (Full Rep) 2014;218:1-219. doi:10.23970/ahrqepcerta218

5 Kolodny A, Courtwright DT, Hwang CS, et al. The prescription opioid and heroin crisis: a public health approach to an epidemic of addiction. Annu Rev Public Health 2015;36:559-74. doi:10.1146/ annurev-publhealth-031914-122957

6 Compton WM, Volkow ND. Abuse of prescription drugs and the risk of addiction. Drug Alcohol Depend 2006;83(Suppl 1):S4-7. doi:10.1016/j.drugalcdep.2005.10.020

7 Darnall BD, Stacey BR, Chou R. Medical and psychological risks and consequences of long-term opioid therapy in women. Pain Med 2012;13:1181-211. doi:10.1111/j.1526-4637.2012.01467.x

8 Edlund MJ, Martin BC, Russo JE, DeVries A, Braden JB, Sullivan $M D$. The role of opioid prescription in incident opioid abuse and dependence among individuals with chronic noncancer pain: the role of opioid prescription. Clin J Pain 2014;30:557-64. doi:10.1097/ AJP.0000000000000021

9 Edlund MJ, Sullivan M, Steffick D, Harris KM, Wells KB. Do users of regularly prescribed opioids have higher rates of substance use problems than nonusers?Pain Med 2007;8:647-56. doi:10.1111/ j.1526-4637.2006.00200.x

10 Jalal H, Buchanich IM, Roberts MS, Balmert LC, Zhang K, Burke DS Changing dynamics of the drug overdose epidemic in the United States from 1979 through 2016. Science 2018;361:eaau1184. doi:10.1126/science.aau1184

11 Bohnert ASB, Guy GPJr, Losby JL. Opioid prescribing in the United States before and after the Centers for Disease Control and Prevention's 2016 opioid guideline. Ann Intern Med 2018;169:36775. doi:10.7326/M18-1243

12 Lin LA, Bohnert ASB, Kerns RD, Clay MA, Ganoczy D, Ilgen MA. Impact of the Opioid Safety Initiative on opioid-related prescribing in veterans. Pain 2017;158:833-9. doi:10.1097/j. pain.0000000000000837

13 Soelberg CD, Brown REJr, Du Vivier D, Meyer JE, Ramachandran BK. The US opioid crisis: current federal and state legal issues. Anesth Analg 2017;125:1675-81. doi:10.1213/ANE.0000000000002403

14 CDC Injury Center. Opioid overdose: CDC guideline for prescribing opioids for chronic pain. https://www.cdc.gov/drugoverdose/ prescribing/guideline.html. 2019

15 National Academies of Sciences, Engineering, and Medicine. Pain management and the opioid epidemic: balancing societal and individual benefits and risks of prescription opioid use. Washington, DC: National Academies Press, 2017. https://doi. org/10.17226/24781

16 Dowell D, Haegerich TM, Chou R. CDC Guideline for prescribing opioids for chronic pain--United States, 2016. JAMA 2016;315:1624-45. doi:10.1001/jama.2016.1464

17 Chiu AS, Jean RA, Hoag JR, Freedman-Weiss M, Healy JM, Pei KY. Association of lowering default pill counts in electronic medical record systems with postoperative opioid prescribing. JAMA Surg 2018;153:1012-9. doi:10.1001/jamasurg.2018.2083

18 Zivin K, White JO, Chao S, et al. Implementing electronic health record default settings to reduce opioid overprescribing: a pilot study. Pain Med 2019;20:103-12. doi:10.1093/pm/pnx304

19 Delgado MK, Shofer FS, Patel MS, et al. Association between electronic medical record implementation of default opioid prescription quantities and prescribing behavior in two emergency departments. J Gen Intern Med 2018;33:409-11. doi:10.1007/ s11606-017-4286-5

20 Blutinger EJ, Shofer FS, Meisel Z, Perrone J, Engel-Rebitzer E, Delgado MK. Variability in emergency department electronic medical record default opioid quantities: a national survey. Am J Emerg Med 2019;66:3. doi:10.1016/j.ajem.2019.03.023

21 Chang HY, Murimi I, Faul M, Rutkow L, Alexander GC. Impact of Florida's prescription drug monitoring program and pill mill law on high-risk patients: a comparative interrupted time series analysis. Pharmacoepidemiol Drug Saf 2018;27:422-9. doi:10.1002/ pds. 4404

22 Lyapustina T, Rutkow L, Chang H-Y, et al. Effect of a "pill mill" law on opioid prescribing and utilization: the case of Texas. Drug Alcohol Depend 2016;159:190-7. doi:10.1016/j.drugalcdep.2015.12.025

23 Kennedy-Hendricks A, Richey M, McGinty EE, Stuart EA, Barry CL, Webster DW. Opioid overdose deaths and Florida's crackdown on pill mills. Am J Public Health 2016;106:291-7. doi:10.2105/ AJPH.2015.302953

24 Rutkow L, Chang H-Y, Daubresse M, Webster DW, Stuart EA Alexander GC. Effect of Florida's prescription drug monitoring program and pill mill laws on opioid prescribing and use. JAMA Intern Med 2015;175:1642-9. doi:10.1001/jamainternmed.2015.3931

25 Chang H-Y, Lyapustina T, Rutkow L, et al. Impact of prescription drug monitoring programs and pill mill laws on high-risk 
opioid prescribers: a comparative interrupted time series analysis. Drug Alcohol Depend 2016;165:1-8. doi:10.1016/j. drugalcdep.2016.04.033

26 Betses M, Brennan T. Abusive prescribing of controlled substances-a pharmacy view. N Engl J Med 2013;369:989-91. doi:10.1056/ NEJMp1308222

27 Sacarny A, Yokum D, Finkelstein A, Agrawal S. Medicare letters to curb overprescribing of controlled substances had no detectable effect on providers. Health Aff (Millwood) 2016;35:471-9. doi:10.1377/ hlthaff.2015.1025

28 Doctor JN, Nguyen A, Lev R, et al. Opioid prescribing decreases after learning of a patient's fatal overdose. Science 2018;361:588-90. doi:10.1126/science.aat4595

29 Dowell D, Haegerich T, Chou R. No shortcuts to safer opioid prescribing. N Engl J Med 2019;380:2285-7. doi:10.1056/NEJMp1904190

30 Chen JH, Humphreys K, Shah NH, Lembke A. Distribution of opioids by different types of medicare prescribers. JAMA Intern Med 2016;176:259-61. doi:10.1001/jamainternmed.2015.6662

31 Swedlow A, Ireland J, Johnson G. Prescribing patterns of schedule II opioids in California workers' compensation. California Workers' Compensation Institute; 2011. https://www.cwci.org/document. php?file=1438.pdf.

32 National Center for Injury Prevention and Control. CDC compilation of benzodiazepines, muscle relaxants, stimulants, zolpidem, and opioid analgesics with oral morphine milligram equivalent conversion factors, 2017 version. Centers for Disease Control and Prevention; 2017. https://www.cdc.gov/drugoverdose/resources/data.html.

33 Reed WJ. The Pareto, Zipf and other power laws. Econ Lett 2001:74:15-9 doi:10.1016/S0165-1765(01)00524-9

34 Barnett ML, Olenski AR, Jena AB. Opioid-prescribing patterns of emergency physicians and risk of long-term use. N Engl J Med 2017:376:663-73 doi:10.1056/NEIMsa1610524

35 Schieber LZ, Guy GPJr, Seth P, et al. Trends and patterns of geographic variation in opioid prescribing practices by state, United States, 2006-2017. JAMA Netw Open 2019;2:e190665. doi:10.1001 jamanetworkopen.2019.0665

36 Guy GPJr, Zhang K. Opioid prescribing by specialty and volume in the U.S. Am J Prev Med 2018;55:e153-5. doi:10.1016/j. amepre.2018.06.008

37 Levy B, Paulozzi L, Mack KA, Jones CM. Trends in opioid analgesicprescribing rates by specialty, U.S., 2007-2012. Am J Prev Med 2015:49:409-13. doi:10.1016/i.amepre.2015.02.020

38 Hoffman KM, Trawalter S, Axt JR, Oliver MN. Racial bias in pain assessment and treatment recommendations, and false beliefs about biological differences between blacks and whites. Proc Natl Acad Sci U S A 2016;113:4296-301. doi:10.1073/pnas.1516047113

39 MDCalc. Benzodiazepine conversion calculator. https://www.mdcalc. com/benzodiazepine-conversion-calculator

40 DeCarolis DD, Rice KL, Ho L, Willenbring ML, Cassaro S. Symptomdriven lorazepam protocol for treatment of severe alcohol withdrawal delirium in the intensive care unit. Pharmacotherapy 2007;27:510-8 doi:10.1592/phco.27.4.510

41 Latt N, Conigrave K, Saunders JB, Marshall JE, Nutt D, eds. Addiction medicine. Oxford University Press, 2009. doi:10.1093/ med/9780199539338.003.0003

42 Tyrer P, Silk KR, eds. Cambridge textbook of effective treatments in psychiatry. Cambridge University Press, 2008:3-15. doi:10.1017| cb09780511544392.002

43 Gelenberg AJ, Keith S, eds. The practitioner's guide to psychoactive drugs . Springer US, 1998:153-212. doi:10.1007/978-1-46155877-4_4.

44 Gini C. Measurement of inequality of incomes. The Economic Journal. 1921. https://www.jstor.org/stable/2223319.

45 Mills JA, Zandvakili S. Statistical inference via bootstrapping for measures of inequality. J Appl Econ 1997. doi:10.1002/(SICI)10991255(199703)12:2«133::AID-JAE433»3.0.CO;2-H.

46 AHRQ Agency for Healthcare Research and Quality. HCUP-US Tools \& Software. https://www.hcup-us.ahrq.gov/tools_software.jsp

47 Cowell FA. Measuring inequality. London School of Economics perspectives in economic analysis. Oxford Scholarship Online, 2011:1-16. doi:10.1093/acprof:osobl/9780199594030.003.0001

48 United Nation's International Narcotics Control Board. Progress in ensuring adequate access to internationally controlled substances for medical and scientific purposes: supplement to the report of the board for 2018. https://doi.org/10.18356/9b973caa-en.

49 Kiang MV, Basu S, Chen J, Alexander MJ. Assessment of changes in the geographical distribution of opioid-related mortality across the United States by opioid type, 19992016. JAMA Netw Open 2019;2:e190040. doi:10.1001/ jamanetworkopen.2019.0040

50 Martin J, Cunliffe J, Décary-Hétu D, Aldridge J. Effect of restricting the legal supply of prescription opioids on buying through online illicit marketplaces: interrupted time series analysis. BM/2018:361:k2270. doi:10.1136/bmj.k2270

\section{Web appendix: Supplementary materials}

